\title{
Mobile Agents based Reliable and Energy Efficient Routing Protocol for MANET
}

\author{
Saravanan Nallusamy ${ }^{1 *}$, Subramani Appavupillai' ${ }^{2}$, Sivakumar Ponnusamy ${ }^{3}$ \\ ${ }^{1}$ K. S. R. College of Engineering, Tiruchengode, Namakkal, Tamilnadu, India \\ ${ }^{2}$ Government Arts College, Dharmapuri, Tamilnadu, India \\ ${ }^{3}$ K. S. R College of Engineering, Tiruchengode, Tamilnadu, India \\ *Corresponding author's Email: nsaravanan0678@gmail.com
}

\begin{abstract}
In this paper, we propose Mobile Agent based Energy Efficient Reliable routing protocol for MANET. At first the protocol uses a link cost metric such as Network Load in terms of node burthen degree and bandwidth usable degree, Minimum Drain Rate (MDR) for energy consumption and Link availability. The mobile agents are randomly organized and transfer hop by hop until the destination is reached. Hence, from each hop they traverse, they collect the information related to the above metrics and a combined list cost metric is estimated based on these metrics. Finally, after collecting information's from agents multiple paths are established first and then the source selects the optimal path using the path cost metric, which is the summation of link cost metric along the path.
\end{abstract}

Keywords: MANET; Minimum Drain Rate; topology; Routing protocol.

\section{Introduction}

\subsection{MANET}

A MANET is an autonomous collection of nodes mobile users that offers infrastructure-free architecture for communication over a shared wireless. Mobile Ad-Hoc Network (MANET) is a self-configuring network of mobile nodes connected by wireless links forming a random topology. The nodes are free to move arbitrarily. Thus, the network's wireless topology may be random and may change quickly. The network operates in an individual manner that linked to the larger internet. An Ad-hoc network is formed by sensor networks consisting of sensing, data processing and communication components. Due to incomplete infrastructure support, each node acts as a router, forwarding data packets for other nodes. MANET nodes have limited processing speed and power, battery, storage, and communication capabilities. One of the most challenging issues in MANETs is their routing algorithms $[1,2,3]$.

\section{Issues}

- Error-prone channel state

- Bandwidth-constrained

- Energy-constrained operation

- Security Issues [1, 4]

\section{Limitations}

- Processing speed

- Power

- Battery

- Storage

- Communication capabilities $[1,3]$

\subsection{Energy Efficient Routing in MANET}

Routing protocols in MANETs are classified under two major fields of protocols:

- Proactive or table-driven

- Reactive or on-demand [5]

The efficient node-energy utilization in mobile ad-hoc networks is an essential role. Death of node due to energy exhausted in ad hoc network leads to the network partition and causes communication failure in the network [7]. Since energy is limited in wireless Mobile Ad-hoc Networks, designing energy 
aware routing protocols has become a main issue. The aim of these protocols is to reduce the energy consumption of the mobile nodes in the network in order to maximize the lifetime of the network [5]. The Minimum Energy routing protocols can be further divided into three classes based on the types of link costs:

- Minimum Total Transmission Power (MTTP),

- Minimum Total Transceiving Power (MTTCP),

- Minimum Total Reliable Transmission Power (MTRTP) [3] [12]

\section{Issues}

- Unpredictability of environment

- Unreliability of wireless medium

- Resource-constrained nodes $[6,7]$

\subsection{Reliable in MANET}

The most critical network resource is considered as bandwidth, although power efficiency is also of prime concern. The different approaches used different parameters to achieve a reliable route like node residual energy, link expiration time, link available time, stability of nodes, node successful data transmission, received signal strength etc.

\section{Issues}

- Link quality

- Load balancing $[8,10]$

In this paper to develop Mobile Agent, based Energy Efficient Reliable routing protocol for MANET link cost metric is designed based on the following metrics in this protocol are Network Load in terms of node burthen degree and bandwidth usable degree [13], Minimum Drain Rate (MDR) for energy consumption [7], Link availability [11]. From each hop they traverse, they collect the information related to the above metrics and a combined list cost metric is estimated based on these metrics. After collecting information from the agents, multiple paths are established first and then the source selects the optimal path using the path cost metric, which is the summation of link cost metric [14] along the path.

\section{Related Works}

\subsection{Energy Efficient Routing in MANET}

S. Jamali et al. [1] have proposed the Binary Particle Swarm Optimization algorithm (BPSO) to add the energy awareness feature to the TORA routing protocol. The protocol considers routes length in its route selection process and includes routes energy level in its calculations. They have formulated the routing issue as an optimization problem and then employ BPSO to choose a route that maximizes a weighted function of the route length and the route energy level.

J. Zhu and X. Wang [3] have presented that the minimum energy routing schemes in the fails without considering the routing overhead involved and node mobility. They have been developing a more accurate analytical model to track the energy consumptions due to various factors, and a simple energy-efficient routing scheme PEER to improve the performance during path discovery and in mobility scenarios.

M. C. Aye and A. M. Aung [5] have proposed an energy efficient multipath routing protocol for selection of an energy efficient way. The system considers transmission power of nodes and residual energy as energy metrics in order to maximize the network lifetime and to reduce energy consumption of mobile nodes. The system was used to find an optimal route based on two energy metrics while choosing a route to transfer data packets.

H. Xiao et al. [6] have proposed a set of performance metrics in evaluating energy efficiency in MANETs, and the energy consumption of MANET from a variety of aspects: at different network layers including application layer, network layer and MAC layer, at different operation mode including idle, transmit and receive, and with different routing protocols including DSR, DSDV and AODV.

F. D. Rango et al. [7] have proposed an approach that tries to account for link stability and for minimum drain rate energy consumption. Hence to verify the correctness of the solution bi-objective optimization formulations have been developed and a routing protocol called LAER (Link-stability and Energy Aware Routing protocols) was used. The schemes have been compared with other three protocols: PERRA, GPSR and E-GPSR.

\subsection{Reliability in MANET}

A. Barolli et al. [8] have proposed a Genetic Algorithms (GAs) and Multi-Objective Optimization for QoS routing in Ad-hoc Networks. Furthermore, to reduce the search space of GA, was implemented a Search Space Reduction Algorithm (SSRA). After the reduction of search space the GAMAN, search time improves. 
L. Hanzo et al. [9] have proposed and evaluated solutions for improving the performance of QAR and $\mathrm{AC}$ protocols in the face of mobility, shadowing and varying link SINR. It was found that proactively maintaining backup routes for active sessions, adapting transmission rates, and routing around temporarily low-SINR links could noticeably improve the reliability of assured throughput services.

L. C. Llewellyn et al. [10] have presented a cluster-based QoS routing algorithm for mobile ad hoc for fault tolerance, which was a critical feature in providing QoS in the link failure-prone environment of mobile networks. Furthermore, the fault-tolerant cluster-based QoS wireless algorithm was evaluated according to failure recovery time, dropped packets, throughput and sustained flow bandwidth involving the node failure scenarios along QoS paths.

R. Prabha and Ramaraj [11] have proposed Ad hoc On-demand Multipath Distance Vector (AOMDV) based on link availability, neighboring node's queuing delay, node mobility and bit error rate. The optimal path was selected using BAT meta-heuristic optimization.

\section{Problem Identification}

From the literature survey of the above two category of existing works, we identify the following problems:

In existing energy efficient routing protocols,

- Minimum drain rate energy consumption is not considered

- Network load balancing is not considered in existing reliable routing protocols,

- Link availability and queuing delay are not considered.

- Routing overhead is not reduced.

Though there are lot of works done on energy efficiency and reliability, none of the work focus on both.

Hence, our objective is to design an energy efficient reliable routing, which solves the above issues.

\section{Proposed Method}

To develop Mobile Agent based Energy Efficient Reliable routing protocol for MANET a link cost metric is designed

- At first link, cost metric is designed

- Then the mobile agents are randomly deployed over the source nodes and migrate hop by hop until the destination is reached
- From each hop they traverse, they collect the information related to the above metrics and a combined list cost metric is estimated based on these metrics.

- After collecting information from the agents, multiple paths are established first and then the source selects the optimal path using the path cost metric, which is the summation of link cost metric [14] along the path.

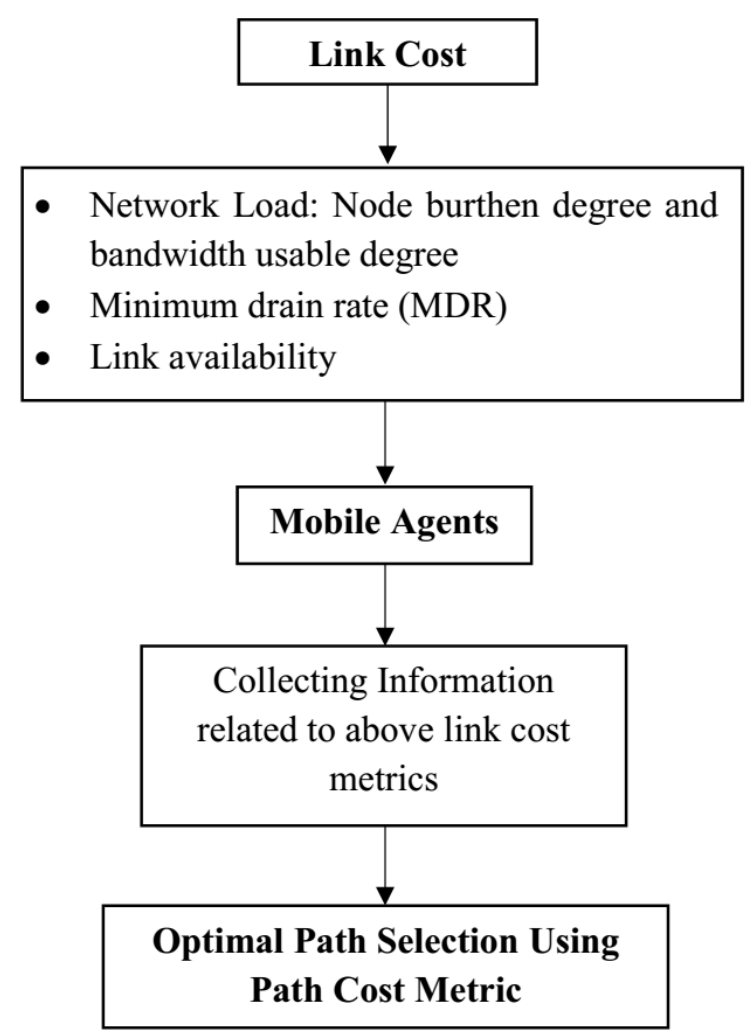

Figure.1 Block Diagram of the proposed method

\subsection{Link Cost Metrics}

The link cost metrics is estimated using the following metrics such as network load, Minimum Drain Rate and link availability. It is as follows

\subsubsection{Network Load}

The network load works based on node burthen degree and bandwidth usable degree. Hence, look in to how the node burthen degree and bandwidth usable degree of network load

- Node Burthen Degree

$$
N B D=\frac{u_{x}+\sum_{y \in N} u_{x}^{y}}{C_{x}+\sum_{y \in N} C_{x}^{y}}
$$


Where,

$u_{x}$ - Queue length of the node $\mathrm{x}$

$u_{x}^{y}$ - Queue length of the node which is neighbor to the node $\mathrm{x}$

$C_{x}$ - Cache length of the node $\mathrm{x}$

$C_{x}^{y}$ - Cache length of the node which is neighbor to the node $\mathrm{x}$

\section{- Bandwidth Usable Degree}

$$
B U D=\frac{A_{b}}{N_{b}}
$$

Where,

BUD- bandwidth usable degree

$A_{b}$ - Available bandwidth of the node

$N_{b}$ - Total bandwidth of the node

\subsubsection{Minimum Drain Rate}

In Minimum Drain Rate, the node will accept all route requests only if it has enough residual battery capacity. Due to this logic, the drain rate of energy consumption will rise to high and resultant in fall of battery. To overcome the problem, traffic loan characteristics has to be evaluated. At first the node $\mathrm{x}$ will observe the energy consumption caused by the transmission, response and overhearing behavior and then evaluate the drain rate denoted as $R D_{x}$ for every seconds and it is denoted as T. The $R D_{x}$ is represented as follows

$$
\begin{gathered}
R D_{c r, x}=R D_{x}(t) \\
R D_{x}(t)=\beta \cdot R D_{x}(t-1)+(1-\beta) \cdot R D_{c r, x}
\end{gathered}
$$

Where,

$R D_{c r, x}$ - Previous and new calculated rates

\subsubsection{Link Availability}

The link availability between two nodes for a particular duration, the node velocity and the distance between the nodes less than the minimum transmission range of the node is evaluated as follows,

$$
L A\left(D_{t}\right)=L A_{1}\left(D_{t 1}\right)+\left(L A_{2}\left(D_{t 2}\right)\right.
$$

Where,

$L A$-Link Availability
$D_{t}$ - Duration of link availability between two nodes

The direction of node movement, the direction of node that moves towards or away from each other can be found out using Received Signal Strength Indication (RSSI). If both nodes are moving away from each other means then the link quality can be evaluated as

$$
L A(w)=\frac{w+\left(D_{t}-w\right)}{D_{t}}
$$

\subsection{Mobile Agents}

In Mobile Agent (MA) every node has their own routing table in which it stores the $\mathrm{N}$ new routing information records from all i.e. each and every node such as

Rs: [Rs, $\{(\mathrm{Tx}, \mathrm{Nhx}, \mathrm{Anx}$, and Nx $) \ldots(\mathrm{Tm}, \mathrm{Nhm}$, Anm, Nm)\}]

Where,

Tx - Time of visiting the adjacent node Ax

Nhx - Number of hops

$\mathrm{Nx}$ - Number of MAs on Ax

MA- Mobile Agent

Hence then MA visits a node $\mathrm{N}$, then the routing information of the node $\mathrm{N}$ is updated.

\subsection{Optimal Path Selection}

After collecting information's from agents, multiple paths are established first and then the source selects the optimal path using the path cost metric, which is the summation of link cost metric along the path. The optimal path selection using link cost metric function is as follows:

$$
\begin{gathered}
O L_{C}=\sum\left(N L+R D_{x}+L A\left(D_{t}\right)\right) \\
N L=(N B D+B U D)
\end{gathered}
$$

By applying Network Load (NL) such as NBD (eqn.1) and BUD (eqn. 2), drain rate (RD) (eqn. 4) and Link availability (LA) (eqn.5) in equation (7), we get

$$
\begin{aligned}
O L_{C}=\sum & \left(\left(\frac{u_{x}+\sum_{y \in N} u_{x}^{y}}{C_{x}+\sum_{y \in N} C_{x}^{y}}\right)+\left(\frac{A_{b}}{N_{b}}\right)\right)+ \\
& \left(\beta \cdot R D_{x}(t-1)+(1-\beta) \cdot R D_{c r, x}\right)+ \\
& \left(L A_{1}\left(D_{t 1}\right)+\left(L A_{2}\left(D_{t 2}\right)\right)\right.
\end{aligned}
$$

Where,

$N L$ - Network Load

$N B D$ - Node Burthen degree

$B U D$ - Bandwidth usable degree

$R D_{x}$ - Drain Rate 
$L A\left(D_{t}\right)$-Link Availability

\section{Overall Algorithm}

The overall algorithm for developing Mobile Agent based Energy Efficient Reliable routing protocol for MANET is shown below,

- A link cost metric is designed based on Network Load in terms of node burthen degree and bandwidth usable degree, Minimum Drain Rate (MDR) for energy consumption and Link availability

- Mobile agents are employed after designing the link cost metrics

- They collect information's related to the link cost metrics and estimation for the same is carried out

- After collecting information from the agents, multiple paths are established

- Then the source selects the optimal path using the path cost metric

\section{Simulation Results}

\subsection{Simulation Parameters}

We use NS2 [16] to simulate our proposed Mobile Agents based Reliable and Energy Efficient Routing Protocol (MAREERP). We use the IEEE 802.11 for MANETs as the MAC layer protocol. It has the functionality to notify the network layer about link breakage. In our simulation, the flows are varied as 2, 4, 6 and 8 . The area size is 1000 -meter $x$ 1000-meter square region for 50 seconds simulation time. The simulated traffic is Constant Bit Rate (CBR).

Our simulation settings and parameters are summarized in table 1.

Table 1. Simulation parameters

\begin{tabular}{|l|l|}
\hline No. of Nodes & 50 \\
\hline Area & 1000 X 1000 \\
\hline MAC & 802.11 \\
\hline Simulation Time & $50 \mathrm{sec}$ \\
\hline Traffic Source & CBR \\
\hline Rate & $100 \mathrm{~Kb}$ \\
\hline Propagation & Two Ray Ground \\
\hline Antenna & Omni Antenna \\
\hline Pause Time & $5,10,15,20$ and 25 \\
\hline Flows & $2,4,6$ and 8 \\
\hline
\end{tabular}

We evaluate performance of the new protocol mainly according to the following parameters. We compare the AMRA [14] protocol with our proposed MAREERP protocol.

Average Packet Delivery Ratio: It is the ratio of the number of packets received successfully and the total number of packets transmitted.

Average end-to-end delay: The end-to-enddelay is averaged over all surviving data packets from the sources to the destinations.

Throughput: The throughput is the amount of data that can be sent from the sources to the destination.

Packet Drop: It is the number of packets dropped during the data transmission

\subsection{Results \& Analysis}

The simulation results are presented in the next section.

\section{A. Based on Flows}

In our first experiment, we are varying the number of flows as 2, 4, 6 and 8 for CBR traffic.

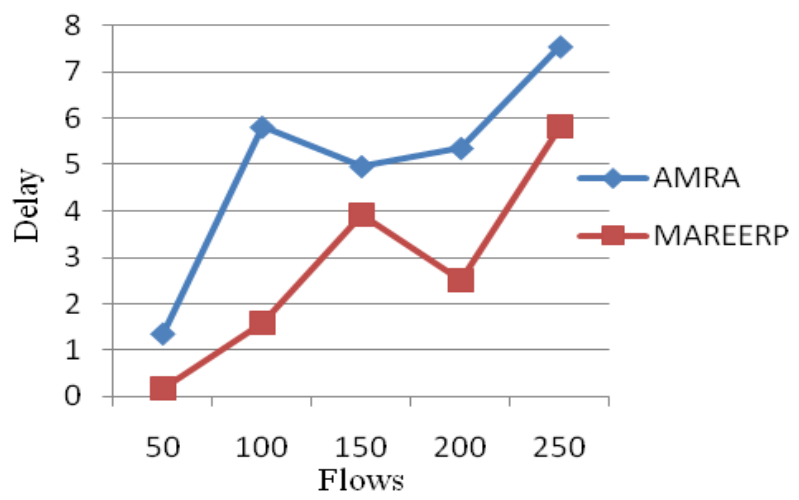

Figure.2 Flows vs. Delay

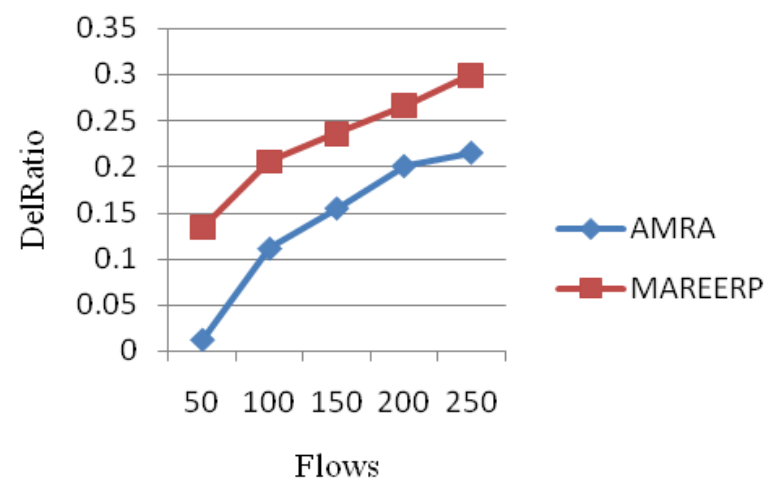

Figure.3 Flows vs. Delivery Ratio

\subsection{Performance Metrics}




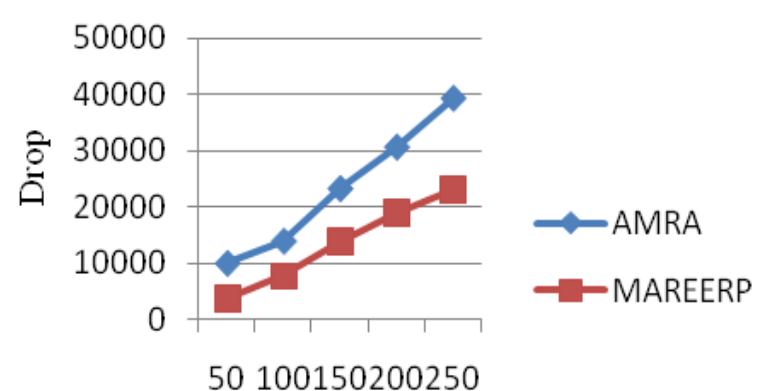

Flows

Figure.4 Flows vs. Drop

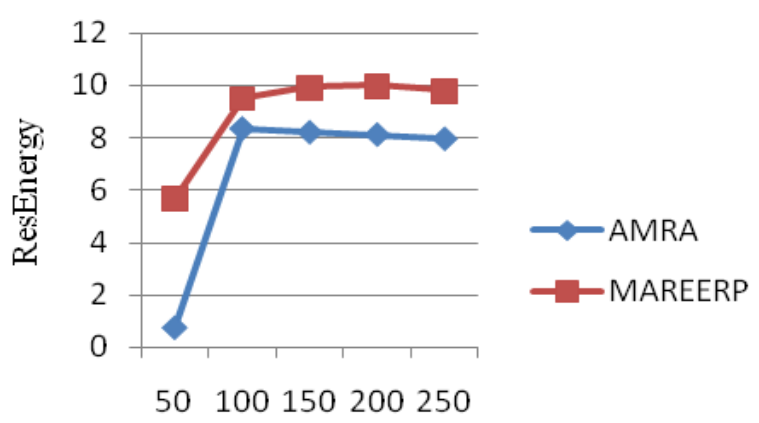

Flows

Figure.5 Flows vs. Residual Energy

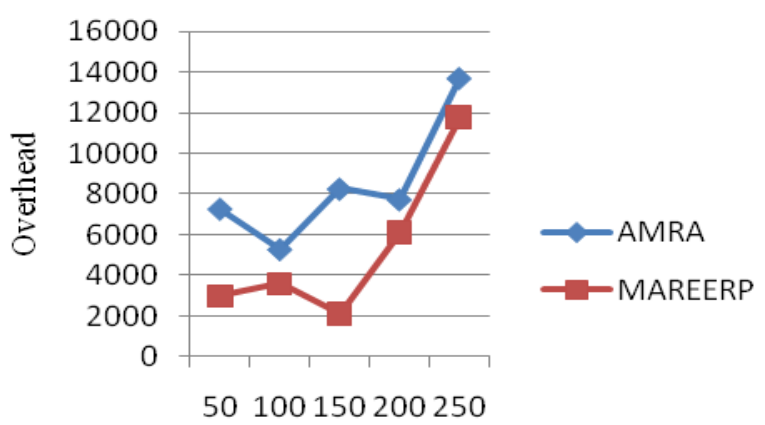

Flows

Figure.6 Flows vs. Overhead

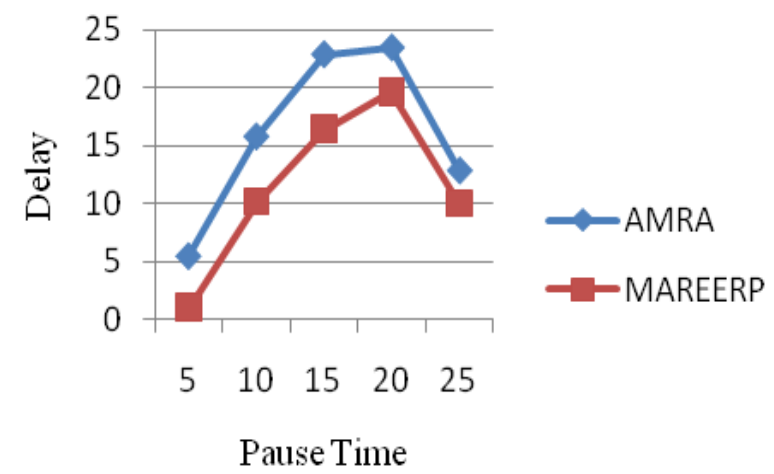

Figure.7 Pause Time vs. Delay
Figures 2 to 6 show the results of delay, delivery ratio, packet drop, residual energy and overhead by varying the flows from 2 to 8 for the CBR traffic in MAREERP and AMRA protocols. When comparing the performance of the two protocols, we infer that MAREERP outperforms AMRA by $42 \%$ in terms of delay, $55 \%$ in terms of delivery ratio, $66 \%$ in terms of packet drop, $12 \%$ in terms of residual energy and $53 \%$ in terms of overhead.

\section{B. Based on Pause Time}

In our second experiment, we are varying the Pause Time as 5,10,15,20 and 25 secs for CBR traffic.

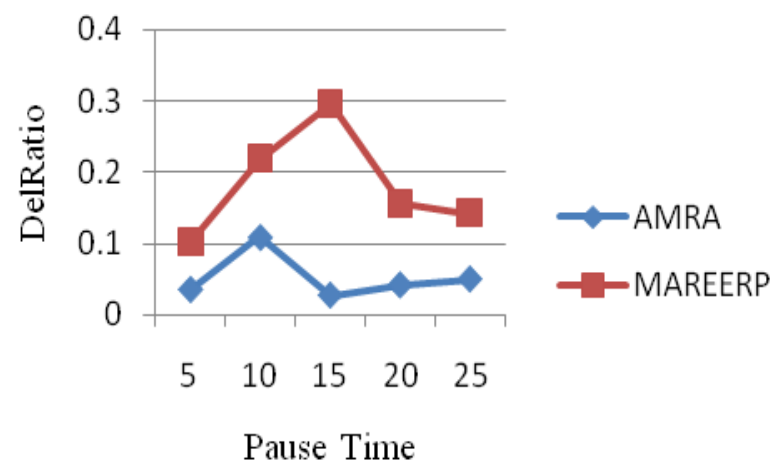

Figure. 8 Pause Time vs. Delviery Ratio

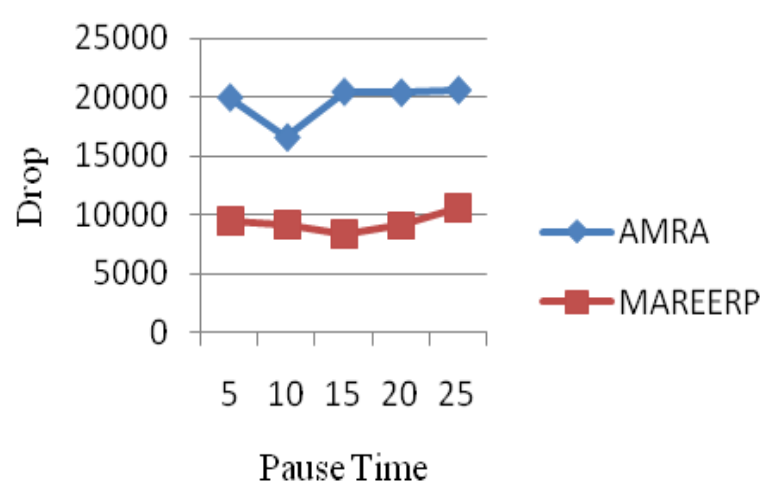

Figure.9 Pause Time vs. Drop

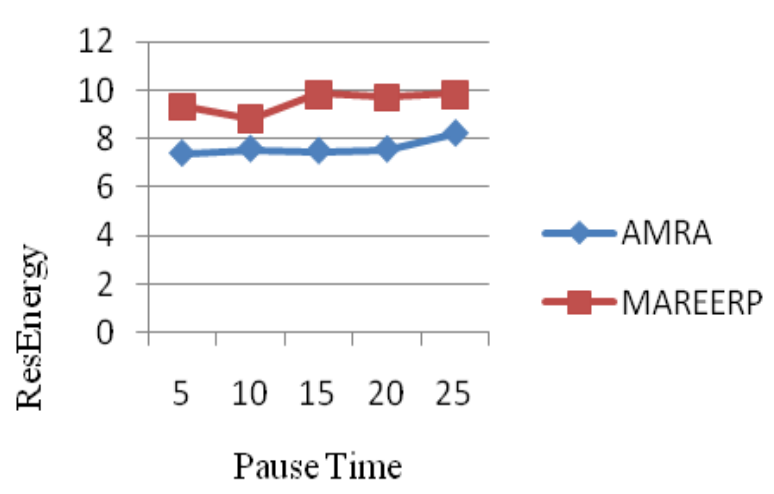

Figure.10 Pause Time vs. Residual Energy 


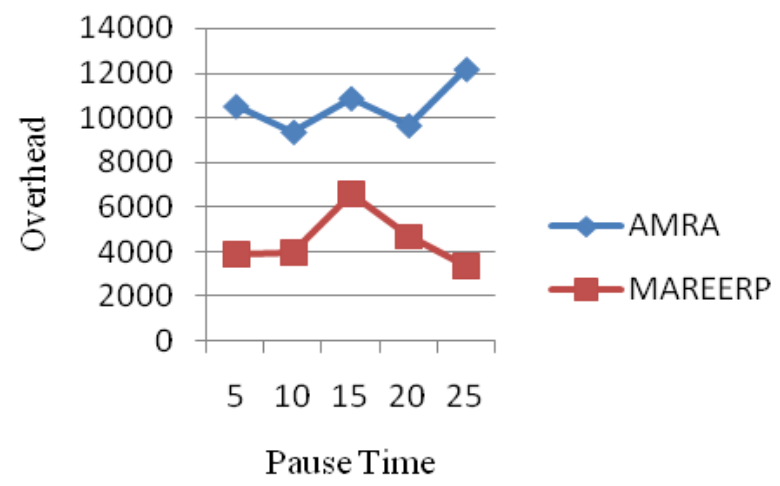

Figure.11 Pause Time vs. Overhead

Figures 7 to 11 show the results of delay, delivery ratio, packet drop, residual energy and overhead by varying the Pause Time from 5 to 25 sec for the CBR traffic in MAREERP and AMRA protocols. When comparing the performance of the two protocols, we infer that MAREERP outperforms AMRA by $18 \%$ in terms of delay, $47 \%$ in terms of delivery ratio, $61 \%$ in terms of packet drop, $8 \%$ in terms of residual energy and 53\% in terms of overhead.

\section{Conclusion}

In this paper a Mobile Agent, based Energy Efficient Reliable routing protocol for MANET is proposed for reliability and energy efficient protocol. The link cost metric is evaluated by means of Network Load in terms of node burthen degree, bandwidth usable degree, Minimum Drain Rate (MDR) for energy consumption and Link availability. Then based on the collected information's a combined list cost metric is estimated. As a final point, after collecting information's from agents multiple paths is established and then the source selected the optimal path using the path cost metric. In future work we have planned to propose adaptive optimization algorithm for routing process that improves the optimal path selection and to improve the system efficiency

\section{References}

[1] S. Jamali, L. Rezaei, and S. J. Gudakahri, "An Energy-efficient Routing Protocol for MANETs: a Particle Swarm Optimization Approach", Journal of Applied Research and Technology, Vol. 11, No. 6, pp. 803-812, 2013.

[2] G. Lee, L. Han, Y. Park, J. B. Lee, J. Kim and H. Peter, "An Energy-Efficient Routing Protocol for CCN-based MANETs", International Journal of Smart Home, Vol. 7, No. 1, pp. 143-152, 2013.
[3] J. Zhu and X. Wang, "Model and Protocol for Energy-Efficient Routing over Mobile Ad Hoc Networks", IEEE Transactions on Mobile Computing, Vol. 10, No. 11, pp. 1546-1557, 2011.

[4] V. N. Talooki, H. marques, and J. Rodriguez, "Energy efficient dynamic MANET on-demand (E2DYMO) routing protocol", IEEE conference of Mobile and Multimedia Networks, pp. 1-5, 2013.

[5] M. C. Aye and A. M. Aung, "Energy Efficient Multipath Routing for Mobile Ad Hoc Networks", International Journal of Information Technology, Modeling and Computing, Vol. 2, No. 3, pp. 11-18, 2014.

[6] H. Xiao, D. M. Ibrahim and B. Christianson, "Energy Consumption in Mobile Ad Hoc Networks", IEEE conference of Wireless Communications and Networking Conference (WCNC), pp. 2599-2604, 2014.

[7] F. D. Rango, F. Guerriero, and P. Fazio, "LinkStability and Energy Aware Routing Protocol in Distributed Wireless Networks", IEEE Transactions on Parallel and Distributed Systems, Vol. 23, No. 4, pp.713-726, 2010.

[8] A. Barolli, E. Spaho, F. Xhafa, L. Barolli and M. Takizawa, "Application of GA and Multi-Objective Optimization for QoS Routing in Ad-hoc Networks", International Conference on Network-Based Information Systems, pp. 50-59, 2011.

[9] L. Hanzo, Member, and R. Tafazolli, "QoS-Aware Routing and Admission Control in Shadow-Fading Environments for Multirate MANETs", IEEE Transactions on Mobile Computing, Vol. 10, No. 5, pp. 622-637, 2011.

[10] L. C. Llewellyn, K. M. Hopkinson, and S. R. Graham, "Distributed Fault-Tolerant Quality of Wireless Networks", IEEE Transactions on Mobile Computing, Vol. 10, No. 2, pp.175-190, 2011.

[11] R. Prabha and Ramaraj, "An improved multipath MANET routingusing link estimation and swarm intelligence", Journal on Wireless Communications and Networking, 2015.

[12] Garcia, Kallel, Kyamakya, Jobmann, Cano, and Manzoni, "A Novel DSR-based Energy-efficient Routing Algorithm for Mobile Ad-hoc Networks", IEEE 58th Conference of Vehicular Technology, Vol. 5, pp. 2849-2854, 2003.

[13] X. Li, and Zhe Li, "LBAMR: A Load Balancing and Asymmetrical Multi-Path Routing", IEEE Transactions on Parallel and Distributed Systems, pp. 1-4, 2009.

[14] S. Kim, "Adaptive MANET Multipath Routing Algorithm Based on the Simulated Annealing Approach", Journal of scientific world, 2014.

[15] V. K. Sharma and Dr. S. S. Bhadauria, "Mobile Agent Based Congestion Control using Aodv Routing Protocol Technique for Mobile Ad-Hoc Network", International Journal of Wireless \& Mobile Networks (IJWMN), Vol. 4, No. 2, pp. 299314, April 2012.

[16] Network Simulator: http:///www.isi.edu/nsnam/ns 